\title{
Volver a la escuela
}

\section{GOING BACK TO SCHOOL}

\section{$\square$ VOLTAR PARA A ESCOLA}

\section{Alejandro Álvarez Gallego* / rizoma.alejandro@gmail.com}

\section{Resumen}

El artículo es un llamado a revisar a fondo los discursos que cuestionan la escuela haciendo eco de las críticas que proliferan desde intereses múltiples, ajenos a las dinámicas propias que se producen en claves que no siempre son fáciles de leer. Se sustenta cómo la escuela es un espacio de igualdad que no existe en ninguna otra institución social contemporánea y que sin ella entraríamos en un vértigo no deseable para los niños y jóvenes que esperan garantías mínimas para su formación. Se advierte sobre el riesgo de caer en posturas conservadoras que desde otras corrientes ideológicas podrían ser cuestionadas; más allá de todo debate teórico o político, la vigencia histórica de la escuela es un asunto que nos remite mas bien a una pregunta ética por la condición humana y por la responsabilidad que los adultos tenemos, en esta cultura, frente a las nuevas generaciones.

\section{Abstract}

The article is a call to thoroughly review discourses that question schools, echoing widespread criticism from the perspective of multiple interests, alien to the very dynamics that are produced in codes not always easy to read. The paper defends that schools constitute a space of equality that does not exist in any other contemporary social institution and without which we would enter in a frenzy, which is undesirable for children and young people who expect minimum guarantees for their education. It warns of the risk of falling into conservative positions that could be questioned from other ideological currents; beyond any theoretical or political debate, the historical validity of the school is an issue that refers us to an ethical question about the human condition and about the responsibility that we adults have, in this culture, regarding new generations.

\section{Resumo}

O artigo é uma chamada para revisar pormenorizadamente os discursos que questionam a escola fazendo eco das críticas que proliferam desde interesses múltiplos, alheios às dinâmicas próprias que são códigos produzidos que nem sempre são fáceis de ler. Afirma-se como a escola é um espaço de igualdade que não existe em nenhuma outra instituição social contemporânea e que sem ela poderíamos entrar em uma vertigem não desejável para as crianças e os jovens que esperam garantias mínimas para sua formação. Adverte-se sobre o risco de cair em posturas conservadoras que desde outras correntes ideológicas poderiam ser questionadas; além de todo debate teórico ou político, a vigência histórica da escola é um assunto que nos remete a uma pergunta ética pela condição humana e pela responsabilidade que os adultos temos, nessa cultura, frente as novas gerações.

\section{Palabras clave}

Escuela; formación; niños y jóvenes; saber pedagógico

\section{Keywords}

School, education, children and young people, pedagogical knowledge.

\section{Palavras chave}

Escola, formação; crianças e jovens; saber pedagógico

\footnotetext{
1 Director Instituto Pedagógico Nacional. Universidad Pedagógica Nacional de Colombia. Grupo de investigación Historia de la práctica pedagógica.
}

Fecha de recepción: 05 de febrero de 2016 / Fecha de aprobación: 09 de mayo de 2016 
Este libro está destinado a lectores tranquilos, a hombres que todavía no se dejen arrastrar por la prisa vertiginosa de nuestra rimbombante época, $y$ que todavía no experimentan un placer idólatra al verse machacados por sus ruedas... o sea, ja pocos hombres! (...) En efecto la cultura auténtica desdeña contaminarse con un individuo necesitado y lleno de deseos: sabe escurrirse astutamente de las manos de quien quiera apoderarse de la cultura como de un medio para sus fines egoístas.

$Y$, cuando alguien cree haberla apresado, para sacar provecho de ella, de algún modo, y, al utilizarla, satisfacer las necesidades de su vida, entonces aquella se escapa súbitamente, con pasos imperceptibles y con actitud desdeñosa".

Friedrich Nietzsche Sobre el porvenir de nuestras escuelas

Este texto pretende plantear un debate con quienes insisten en desprestigiar la escuela, por considerarla un lugar donde se reproducen las clases sociales o un espacio de maltrato y abuso, donde se violan sistemáticamente los derechos de los niños, o, en el mejor de los casos, una institución inflexible y tradicional que no está a tono con los cambios culturales, ni con los avances de las ciencias ni, sobre todo, con las nuevas tecnologías de la comunicación. Hoy es un lugar común escuchar fuertes críticas a la escuela desde las pedagogías Ilamadas alternativas, desde el mundo de la empresa y desde las instituciones del Estado; todas, en procura de reformarla, o, incluso, de suplantarla por otras formas de educar, como la muy reciente modalidad de la educación en casa.

Plantearé, en cambio, que la escuela es un espacio en el cual las nuevas generaciones tienen la oportunidad de vivir unos años protegidas de la incertidumbre y de la velocidad que la sociedad contemporánea propone como un deber ser al que parece inevitable plegarse. Para ello, pondré en relación un texto reciente de Masschelein y Simons (2014), Defensa de la escuela, con mi experiencia como director del Instituto Pedagógico Nacional, el colegio de la Universidad Pedagógica Nacional.

El texto de los autores mencionados es, de verdad, un texto esperanzador. Se me ocurre pensar que es un texto inscrito en un movimiento de inflexión, en un pliegue que se está produciendo en estos tiempos, justo cuando se han instalado en los imaginarios que mueven nuestras prácticas pedagógicas las ideas de la innovación, de la flexibilidad, de la creatividad, de las competencias y del aprender a aprender.

En otro trabajo (Álvarez, 2013), a propósito de la educación popular, me había preguntado cómo ella, junto con la Teología de la liberación, la cibernética de los años sesenta del siglo XX y las luchas contra la reproducción en educación, incluyendo el Movimiento Pedagógico de los maestros colombianos de la década de 1980 (Suárez, 2002), habían funcionado como superficie de emergencia de este momento que hoy reconocemos, con Foucault (2007), como neoliberal ${ }^{1}$. Superficie de emergencia es una manera de decir que, más allá de las relaciones causales y de toda voluntad política o individual, se crean las condiciones para que estos movimientos sean poseídos por una fuerza mayoritaria que la extiende a toda la sociedad y la convierte en una práctica institucionalizada, inevitable y natural.

Por diferentes razones, se nos impele hoy en la escuela a innovar, a cambiar lo que se considera tradicional, memorístico, autoritario, formal, rígido, repetitivo, transmisionista. Quienes participamos de los movimientos de renovación de la escuela durante las décadas de 1970 y 1980 estamos sorprendidos, porque muchas de nuestras reivindicaciones ahora se han convertido en imperativos normativos. Nos sentimos extraños, pues en el transcurso de nuestra propia vida nos cambiaron las banderas de lucha. No nos sentimos contentos con la manera como se han apropiado nuestra causa; hay algo que no nos satisface cuando vemos que el imperativo del cambio se ha convertido en un modo permanente de ser, institucional y personal.

Quiero describir brevemente este momento de transición que vivimos como un momento de incertidumbre, pero también, esperanzador.

\footnotetext{
1 Foucault no entiende el neoliberalismo como un modelo económico, sino como un modo de vida que proviene del momento en que el Estado se gubernamentaliza y da paso al episodio liberal en el que se procura la libertad como principio de la autorregulación. El neoliberalismo sería la radicalización de este episodio, en el cual se vuelve imperativo cambiar la vida, en el sentido de Sloterdijk (2012), a velocidades cada vez más inverosímiles.
} 
¿Qué es lo que no nos gusta? En primer lugar, que sean las grandes corporaciones las que nos ordenan el discurso del cambio; en otro trabajo (Álvarez, 2009) mostraba cómo la publicidad expresa el flujo innovador que atraviesa este momento de la historia, al cual Paolo Virno (2003) ha Ilamado "Multitud"2; dicho autor señalaba en ese trabajo, indagando por lo que le queda hacer a la pedagogía en estos tiempos, que la sociedad de hoy se monta sobre condiciones como la proliferación de juegos lingüísticos y la variación ininterrumpida de las reglas del juego. Los cambios se suceden de manera permanente sobre la base de lo aleatorio y el anonimato; sobre la incertidumbre de expectativas, la contingencia de los puestos y las posiciones, identidades frágiles y valores siempre mutables. En esta sociedad se trata de no tener hábitos, y si se tienen serían como:

- El hábito de moverse de un lado al otro.

- La capacidad de acomodarse a las más bruscas reconversiones.

- La capacidad para adaptarse a cualquier dependencia.

- La ductilidad para cambiar las reglas del juego.

- Manejarse bien en medio de interacciones lingüísticas distintas y banales.

- Manejar diversas alternativas a la hora de tomar una decisión.

Todo esto se aprende en el trabajo y fuera de él. Aunque no se trabaje, la sociedad toda está preparando hoy en esas cualidades. Para comprobarlo, basta con ver los programas de televisión, los realities y los demás programas de concurso, los parques de diversión, los planes turísticos, los deportes de aventura y de alto riesgo; se trata de un proyecto ético que está recogido en la publicidad. Eso es lo que se generaliza hoy por todos los medios, y eso es lo que se le exige a la escuela. Se supone que una escuela renovada y a la altura de los tiempos prepara a los jóvenes para vivir en esa sociedad.

2 En un sentido que no es exactamente igual que el de la Multitud de Hardt y Negri (2004). Sobre esa diferencia no es posible ahondar en el presente artículo, pero, básicamente, se trata de una visión positiva y propositiva que Hardt y Negri le confieren al término, y de una más descriptiva y casi de denuncia que Virno le otorga.
Varios trabajos han mostrado cómo al gran capital le interesa sobremanera esta nueva sociedad del vértigo y la incertidumbre, y eso es lo que en primer lugar nos inquieta. Pero, con Foucault, debemos decir que esto que hoy nos pasa no es resultado de una voluntad política, ni, menos, de unos cambios en la infraestructura económica del capitalismo. En realidad, se trata de cambios capilares, micropolíticos, moleculares, diría Lazzarato (2006), en medio de los cuales todos nos vamos transformando, incluidas las prácticas económicas. Así nos liberamos de buscar culpables o de sentirnos víctimas inmovilizadas por la contundencia de alguna fuerza que estaría manipulando los hilos del poder (como un Gran Hermano). Pero esto no nos deja satisfechos. Hay algo que, en todo caso, nos molesta cuando vemos a los empresarios tan apropiados de un discurso tan flexible.

El segundo malestar es porque esto también lo soñamos nosotros. Cuando nos estaba gustando la posibilidad de inventarnos a nosotros mismos, de hacer de nuestra vida una obra de arte, de pensar un devenir estético de nuestra existencia, de crearnos permanentemente en prácticas de vida cada vez más autogestionadas, al margen del control del Estado, nos encontramos con que ese es un propósito generalizado y que pierde todo su potencial minoritario. Estas consignas las vemos agenciadas, fundamentalmente, en el nuevo discurso de las políticas educativas.

En Colombia hay cuatro líneas de reforma educativa que están moldeando la escuela hacia esa lógica: en primer lugar, la Ley de Ciencia y Tecnología (Ley 1286 de 2009), y que, básicamente,

[...] permite posicionar a la ciencia y la tecnología como base de procesos de innovación conducentes a un modelo económico altamente competitivo [...] la meta a la que se dirigen todos estos esfuerzos es la innovación. La generación, la comunicación y el uso del conocimiento involucra desde los proveedores de bienes y servicios hasta los usuarios y los clientes [...]. (periódico Portafolio, 5 de diciembre de 2008). 
La segunda línea son las directrices del Ministerio de Educación, que busca una escuela para la autogestión, la innovación, las competencias y el aprendizaje. La tercera es la reforma en el Sistema nacional de formación de maestros, el cual busca que las facultades de educación y las escuelas normales formen a docentes competitivos, innovadores, flexibles y creativos (MEN, 2014). La cuarta tiene que ver con el Servicio Nacional de Aprendizaje (SENA), el cual desde la década de 1950 era la institución que formaba para el trabajo en la modalidad de educación no formal, y ahora ya es parte de la educación formal y certifica en competencias laborales para el paso de la educación básica secundaria a la educación técnica y superior. ${ }^{3}$

Dicha reforma comenzó en 2002 y ha tenido una continuidad sorprendente, más allá de los gobiernos, más o menos de derecha, más o menos liberales. Así, hoy están instaladas en nuestras prácticas:

- La gestión escolar, como un modo de autorregularse que tiene la comunidad educativa.

- Las competencias (saber hacer en contexto), que reemplazan los currículos con las áreas del conocimiento convencionales.

- El autoaprendizaje, como un desafío que lleva a cambiar la relación alumno-maestro.

- La evaluación en las pruebas masivas de competencias Ilamadas "Saber", como indicador único de la calidad, determinada en rankings de "mejores" y "peores" colegios.

Inmersos en esta contundente nueva realidad, en Colombia se está generando un movimiento que yo considero hijo de una inflexión, en el sentido que señalé arriba, y al cual podríamos llamar, sin mayores pretensiones, de

3 "Producto de estas acciones es la transmutación del Sistema Educativo al Sistema de Protección Social, lo cual se materializa en el Documento Conpes 3674 del 19 de julio de 2010 y se ratifica en el PND 2010-2014. En su texto se declara conformada la estructura del Sistema de Formación de Capital Humano en Colombia por: a) el Sistema de Educación Formal, b) la Educación para el Trabajo y el Desarrollo Humano y c) el Sistema Nacional de Formación para el Trabajo. Además, se determina que el Sistema de Formación de Capital Humano hace parte del Sistema de Protección Social, entendido este como el sistema que brinda los elementos para proteger a la población frente a riesgos potenciales y vulnerabilidades". (Pinilla, 2011, pp. 7-8) vuelta a la escuela; sería una reacción, no muy consciente, no muy planificada y no muy estructurada, a este afán por innovar a toda costa. Esta inflexión comenzó con la llamada Expedición Pedagógica Nacional (Álvarez, 2001). Dicho proyecto se inició en 1999 y reunió a cerca de 400 maestros de varias regiones del país, con quienes se hicieron visitas a otros colegas (cerca de 3000 experiencias), para reconocer lo que hacen, sin buscar excepcionalidad alguna. Viajamos por el territorio simplemente reconociendo lo que hacen los maestros de manera cotidiana. De estos viajes quedaron diez documentales, más de diez libros y un archivo pedagógico con más de 2000 registros documentales. La mirada expedicionaria de 400 maestros convirtió el trabajo de cerca de 3000 colegas en una experiencia, al reconocerles cómo aún los maestros enseñan, a su manera, a pesar de la guerra, de la pobreza y de las políticas reformistas que buscan convertirlos en simples guías de aprendizajes significativos.

Esta iniciativa expresa una tendencia de muchos colegas, que sin mayores niveles de organización están volviendo a la escuela para ocuparla y ayudar a detener el ritmo vertiginoso de los cambios que la acosan. Volver a la escuela sería un movimiento de maestros que están o han estado en ella y proponen habitarla y reconstruir sus muros poroseados de tantas maneras; incluso, por nosotros mismos. Reconstruir sus muros no significa que la encerremos y la aislemos de lo que pasa hoy en la sociedad; no se trata de eso: se trata de que tengamos tiempo de pensar, de distanciarnos de tantas obviedades y lugares comunes, para que en colectivo, con estudiantes, maestros y familias, reconstruyamos el tejido social y nos juntemos a trabajar como artesanos, con la lentitud propia de quienes quieren hacer las cosas con delicadeza, para disfrutar lo que pasa en la cotidianidad de ese espacio que todavía no ha terminado de diluirse en la incertidumbre del afuera. Este movimiento, no declarado aun como tal, es el que, creo, me ha llevado a dirigir el Instituto Pedagógico Nacional (el colegio de la Universidad Pedagógica).

En el poco tiempo que Ilevo como director, me ha sorprendido la permanencia de viejos rituales que se siguen repitiendo, como el día del arte, el día de las matemáticas y el de la filosofía, la formación de estudiantes, la izada de bandera, la entrega de notas, 
la disciplina, el uniforme, las reuniones de profesores por áreas, los currículos secuenciales por grados; y, por supuesto, las clases, el timbre. En todo esto veo aún mucha alegría, entusiasmo y, por lo tanto, mucha vida. El colorido de las fiestas que se celebran rutina- riamente en el colegio, en rituales que tienen sentido, fundamentalmente, para la escuela y no se repiten en ningún otro escenario, dan cuenta de ello. Las fotos siguientes lo muestran:

\section{Celebración del día del arte en el Instituto Pedagógico Nacional. Bogotá, 25 de septiembre de 2014}
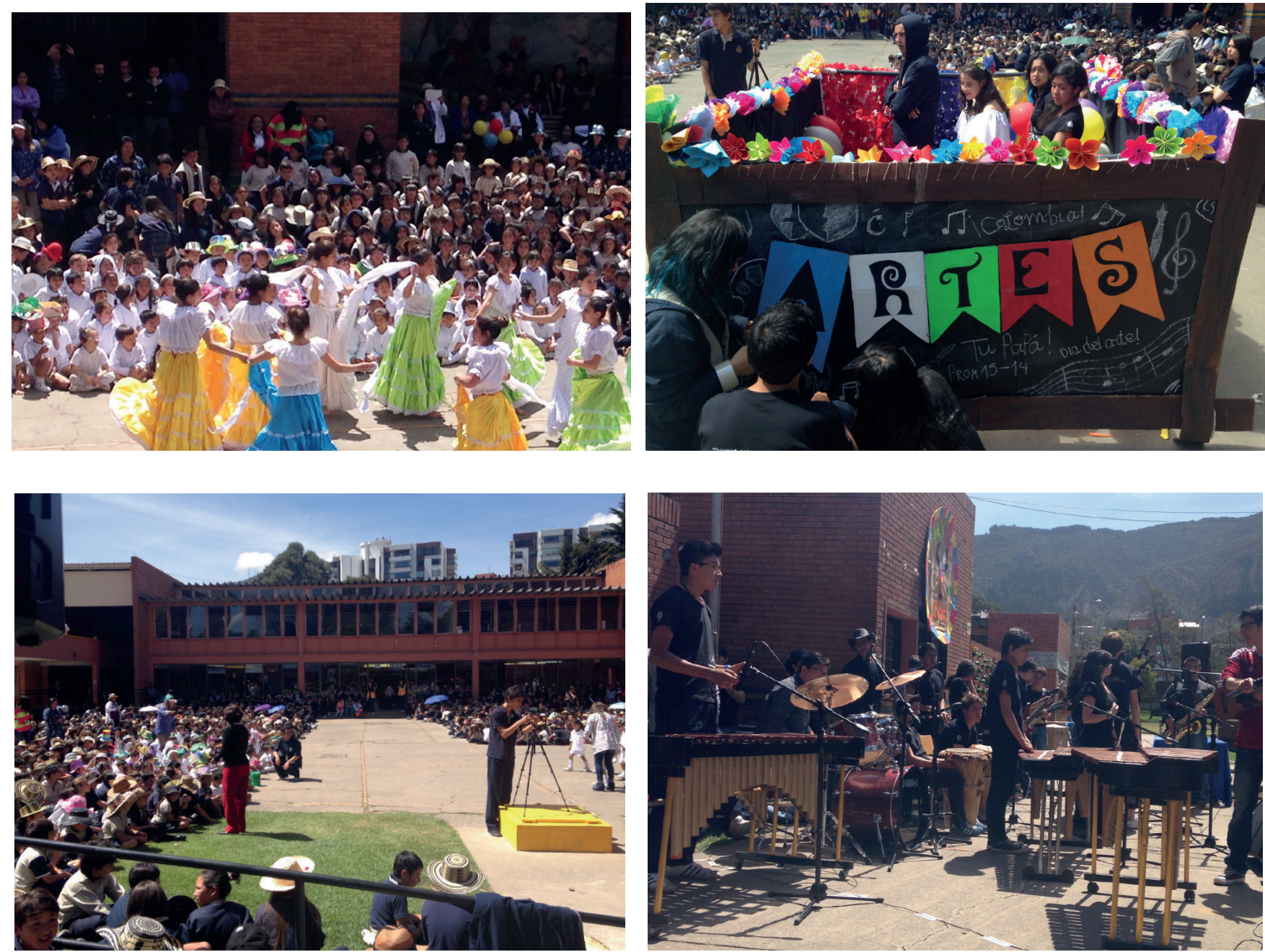

Fuente: fotografía tomada por el autor

La tarea que me he propuesto para los próximos años es indagar con los maestros, los jóvenes y las familias qué tanto de lo que allí sucede vale la pena conservar, potenciar o cambiar; pero con el tiempo que nos da la escuela: el tiempo de los ciclos vitales, de los años de los niños y los adolescentes, de los ritmos de los trimestres académicos, de las celebraciones y de las fiestas escolares.
No voy a negarlo: a veces, percibo en esto que acá he querido llamar "inflexión histórica" un riesgo de asumir posturas conservadoras; pero este miedo hay que enfrentarlo para no renunciar a buscar las fronteras y las fisuras, y para huir de las formas mayoritarias de vida que finalmente nos sujetan, aunque se crea que nos liberan. 
En Colombia hay un movimiento neo-autoritario, Ilamado "Educación al Derecho", el cual promueve un grupo religioso cristiano, con la clara intención de denunciar a los defensores y los promotores de los derechos humanos y los derechos de la infancia en la escuela, pues consideran que esto ha acabado con los valores, con el respeto y con la autoridad que hay que restituir. Acusan al movimiento de renovación pedagógica, que impulsamos durante los decenios de 1980 y 1990, de haber dejado a la escuela a la deriva, de haber producido una pérdida de valores y llevado a la juventud a toda clase de prácticas indeseables. Tal vez, esta veta autoritaria haga parte del mismo proceso de inflexión; por eso, debemos caminar con precaución y sin mayores aspavientos, pues creo que transitamos un terreno poco firme y rodeado de abismos.

Aquí estamos, sin muchas certezas, con muchas intuiciones, buscando cómo hacer de la escuela pública un espacio que sirva para formar a las nuevas generaciones que aún acuden masivamente a ella, pues no se han inventado otro dispositivo que la reemplace. Infortunadamente, en Colombia la educación pública cubre tan solo al $80 \%$ de la población en edad escolar, y existe una brecha infinita entre los privilegios que tienen las élites en sus colegios privados, y la mayoría pobre que se educa en los colegios públicos y en pequeños negocios familiares que ofrecen el servicio en precarias condiciones. De hecho, en los colegios oficiales los niños solo estudian cinco horas (media jornada). El sistema educativo colombiano es, sin duda, uno de los más inequitativos de América Latina (Procuraduría General de la Nación, 2006) y del mundo, y ello es un factor reproductor de la pobreza indiscutible. Es imperativo cambiar esta situación, y para eso se requieren tiempo y una reforma del sistema en su totalidad. Para ello trabajamos de la mano del sindicato de maestros y del movimiento social; pero será un camino para transitar en otra escala.

Por ahora, y mientras tanto, propongo lo que señalé en mis palabras de posesión como director del Instituto Pedagógico Nacional:

"En medio de estas paredes, en medio de sus rituales, del timbre, de las clases, las formaciones, las salidas de campo, los bazares, las notas, las reuniones de padres, las asambleas de profesores, la música, el álgebra, la geografía, la poesía, las voces infantiles y los excesos juveniles, en medio de todo esto que sigue siendo escuela, aunque muchos quieran olvidarlo, acá en estas antiguas costumbres es donde crecerá un puñado de jóvenes que nos empeñamos en formar. Acá un poco a espaldas de tantos afanes por conducir a los niños hacia la sociedad del aprendizaje, a que aprendan toda la vida, a que se globalicen, a que sean innovadores, emprendedores, a que sean productivos, un poco a espaldas de tanto deber ser, se repetirá incesantemente el ritual más simple que fuera inventado hace apenas cuatrocientos años: el del encuentro del maestro con sus alumnos, para enseñarles la humanidad. Acá en este simple pero complejo acontecimiento Ilamado clase, es donde se conserva hoy algo de la genética humana que se resiste a morir en las manos de la guerra, el odio y la injusticia. Acá, en la escuela es uno de los pocos lugares donde aún es posible experimentar la compasión y aprender de ella, donde se puede conversar y discutir para formarnos en la diferencia, donde podemos equivocarnos y recibir una palmada que dice adelante, donde podemos regañarnos y sabemos que no habrá rencores. Esta visión de la escuela, si quieren romántica, es la que considero hoy más potente para resistir el pragmatismo que le exige a la escuela cambiar su gramática del encuentro formativo por la incertidumbre, la que resiste a las presiones para que ya no enseñemos lo que sabemos, para que olvidemos lo que hemos sido. Acá, espero, en medio de las paredes ritualizadas de esta escuela, espero que se siga enseñando mucha historia, mucha aritmética, mucha geometría, mucha poesía y mucha geografía; que la palabra sea respetada en toda su trascendencia y que estos niños y jóvenes puedan vivir una experiencia diferente a la que la sociedad les ofrece, una experiencia en la que vivir no sea un "desafío" o un reality, en la que competir no sea el sentido de sus vidas, una experiencia en la que puedan compartir sin el afán de ser exitosos, innovadores o productivos. Ya habrá tiempo para tener que enfrentarse a ello. Acá esperamos crearles las condiciones para que sepan que vivir no es un reto para superar al otro sino para realizarse en la diferencia; y eso no es poca cosa, tampoco es un camino de rosas, es un inquietante tránsito que la escuela puede acompañar, si no olvida su encargo". 


\section{Referencias}

Álvarez, A. (2001). El viaje y la memoria. Consolidaciones en torno a la Expedición Pedagógica: El riesgo de pensarnos de nuevo. En: Expedición Pedagógica Nacional. Preparando el viaje, Tomo 2, pp. 109-134. Bogotá: Universidad Pedagógica Nacional.

Álvarez, A. (2009). El estatuto teórico de la pedagogía en la transición posmoderna. I Congreso Iberoamericano de Pedagogía. Pedagogía: construcción y transformación en tiempos de incertidumbre. Universidad Nacional de Costa Rica. San José de Costa Rica. Septiembre 29 - 30 y octubre 1.

Álvarez, A. (2013). La autogestión educativa y la gubernamentalidad liberal como superficie de emergencia de la educación popular. Revista Colombiana de Educación, 65, 153-176. Bogotá: Universidad Pedagógica Nacional-CIUP.

Foucault, M. (2007). Nacimiento de la Biopolítica. Buenos Aires: Fondo de Cultura Económica.

Hardt, M. y Negri, A. (2004). Multitud. Barcelona: Debate.

Lazzarato, M. (2006). Políticas del acontecimiento. Buenos Aires: Tinta Limón.
Masschelein, J. y Simons, M. (2014). Defensa de la escuela. Una cuestión pública. Buenos Aires: Miño y Dávila.

Ministerio de Educación Nacional, MEN (2014). Sistema Colombiano de Formación de Educadores y Lineamientos de Política. Bogotá, abril.

Pinilla, P. (2011). El fin de la educación y la deificación de la formación de capital humano. Recuperado de http://www.bdigital. unal.edu.co/7462/1/pedroantoniopinillapacheco.20114.pdf

Procuraduría General de la Nación (2006). El Derecho a la Educación. La Educación en la perspectiva de los Derechos Humanos. Bogotá: Procuraduría General de la Nación.

Sloterdijk, Peter. (2012). Has de cambiar tu vida. Valencia: Catedra.

Suárez, H. (Comp.) (2002). Veinte años del Movimiento Pedagógico. 1982-2002. Entre mitos y realidades. Bogotá: Magisterio, Tercer Milenio.

Virno, P. (2003). Gramática de la Multitud. Madrid: Traficantes de Sueños.

\section{Diálogo del conocimento}

Comparto muchas de las posturas y las preocupaciones que originan el texto de Alejandro Álvarez, "Volver a la escuela". También comparto el entusiasmo por el Manifiesto "En defensa de la escuela" que escribieron Jan Masschelein y Maarten Simons, el cual recoloca la cuestión de lo escolar en coordenadas diferentes de las que se leen habitualmente.

En mi trabajo sobre los vínculos entre la cultura digital y la escuela, arribo a argumentos parecidos. La retórica de las nuevas tecnologías y su poder educativo es una de las más poderosas actualmente; también es una de las más anti-escolares. Vengo llamando la atención sobre la alianza entre las críticas anti-autoritarias de los años 60,70 y 80 y las industrias del edu-business, que quieren convertir lo educativo en otro mercado más. Alianza que alguna vez llamé inesperada, pero también diría que es dolorosa para quienes nos sentimos identificados y fuimos parte de esas luchas. Alejandro Álvarez se refiere a esto con la noción deleuziana de "superficies de emergencia", en las que una fuerza mayoritaria (la retórica educativa de las grandes corporaciones tecnológicas, indistinguible de la de muchas agencias internacionales y de varios ministerios de educación) 'posee' a otros movimientos. Hay que combatir la retórica de la inevitabilidad de lo tecnológico en la educación, sin por eso dejar de reconocer que hay cambios irreversibles; la crítica debería ayudar a desenganchar los medios digitales de muchos de los significantes con que vienen asociados por el $e d u$-business, y a reorganizar las instituciones y las pedagogías en otras direcciones.

"Volver a la escuela" no es volver a la misma institución que criticamos antes, pero sí interrogar las posiciones críticas sobre la escuela como aparato tecnológico-político. Como dice Latour, vivimos en una época iconoclasta, más cerca de quemar monumentos que de erigirlos. Quizás sea más interesante e irreverente preguntarse sobre ese gesto, y suspenderlo por un rato, a ver qué se produce. Parece abrirse un tiempo para pensar con una 
perspectiva que permita desplazarnos de la crítica post-68 a la escuela como la institución disciplinaria total y homogeneizante, y que pueda encontrar, en esa vida y experiencia escolar, formas múltiples. Me parece que la invitación que propone Alejandro, desde su lugar como director del Instituto Pedagógico Nacional, es vital para ese replanteo: "indagar con los maestros, los jóvenes y las familias qué tanto de lo que allí sucede vale la pena conservar, potenciar o cambiar; pero con el tiempo que nos da la escuela: el tiempo de los ciclos vitales, de los años de los niños y los adolescentes, de los ritmos de los trimestres académicos, de las celebraciones y de las fiestas escolares".

La temporalidad, o mejor dicho las múltiples temporalidades, las heterocronías, de las escuelas son un eje central para este "volver a la escuela". Para Simons y Masschelein, la escuela es ante todo una acción de suspensión de un tiempo y creación de un espacio de una cierta duración, o un tiempo "extra" de la cultura. La escuela tiene que ver con crear un tiempo libre, un tiempo no productivo, un tiempo igualitario pero no homogéneo para todos los que están en la escuela, en el que se les propone un tipo específico de trabajo o relación con el saber, un tiempo de estudio, un tiempo de profanación del saber -en el doble sentido de des-sacralizarlo y de volverlo profano, es decir, ponerlo en relación horizontal con las personas-. Este es un acto profundamente democrático, y aunque en sus formas concretas de realización no siempre alcance a todos, la promesa de que puede hacerlo ya instala un horizonte cultural y político diferente al de la visión aristocrática del saber para pocos. Suspendiendo el tiempo y el espacio "actual", creando una posibilidad "inactual" de trabajo con un saber, la institución escolar también permite una cierta atención, un foco, que es la base de ese trabajo con el saber y de ese trabajo con el sí mismo y la comunidad. Es interesante, al respecto, observar la contraposición entre el 'hecho a medida' (tailor-made) de los medios digitales y el 'para todos' de la escuela: hay una tensión que no habría que pasar por alto, ni despachar a la ligera, como solicitaba Benjamin, de las demandas de la historia.

En tiempos tan "anti-escolares", celebro que Alejandro y otros colegas estén, estemos, volviendo a la escuela, no por nostálgicos ni por llevar la contraria, sino porque creemos que hay que defender en ella, de ella, estas posibilidades que abre para una sociedad más democrática. Parafraseando a Ítalo Calvino en "Las ciudades invisibles", es tiempo de encontrar en las escuelas de hoy lo que es "escuela", hacerle espacio y dejar que dure. Enhorabuena.

Inés Dussel 